\title{
IDENTIFICAÇÃO DE SISTEMAS A EVENTOS DISCRETOS MAXPLUS LINEARES
}

\author{
Carlos Andrey Maia* \\ Rafael Santos Mendes ${ }^{\dagger}$ \\ maia@cpdee.ufmg.br \\ rafael@dca.fee.unicamp.br \\ Laurent Hardouin \\ laurent.hardouineistia.univ-angers.fr \\ ${ }^{*}$ Depto. de Engenharia Elétrica - UFMG \\ Av. Antônio Carlos 6627 - Pampulha \\ 31270-010 Belo Horizonte - MG - Brasil \\ ${ }^{\dagger}$ Faculdade de Engenharia Elétrica e de Computação - UNICAMP \\ DCA/FEEC/UNICAMP-C.P. 6101 \\ 13083-970 Campinas - SP - Brasil \\ ${ }^{\ddagger}$ Laboratoire d’Ingénierie des Systèmes Automatisés - ISTIA/Université d'Angers \\ Av. Notre Dame du Lac 62 \\ 49000 Angers - França
}

\section{ABSTRACT}

This paper deals with the identification problem of Max-plus linear Discrete Event Systems with single input and single output. The objective is to estimate the temporal parameters of the system by using the observation of input and output transition firing times assuming that some structural parameters are known. With those assumptions, the identification method is developed by using an upper bound for cycle duration. The paper presents a sufficient excitation condition, concerning the input signal, for the convergence of the algorithm. Examples Illustrate the proposed method.

KEYWORDS: Discrete Event Systems, System Identification, Max-plus Algebra, Timed Event Graphs.

\footnotetext{
ARTIGO CONVIDADO:

Versão completa e revisada de artigo apresentado no CBA-2004

Artigo submetido em 15/12/2004

1a. Revisão em 04/02/2005;

2a. Revisão em 12/04/2005;

Aceito sob recomendação do Ed. Assoc. Prof. Paulo Eigi Miyagi
}

\section{RESUMO}

Este artigo trata do problema de identificação de Sistemas a Eventos Discretos Max-plus lineares mono-entrada e monosaída. O objetivo é estimar os parâmetros temporais do sistema a partir da observação dos instantes de disparo das transições de entrada e de saída, supondo que alguns parâmetros estruturais sejam conhecidos. A partir dessas condições, o método de identificação proposto é desenvolvido tendo como base o cálculo de um limitante superior para o parâmetro de temporização do ciclo. Obtém-se uma condição suficiente de excitação, relativa à variável de entrada, para a convergência do algoritmo proposto. Exemplos ilustram o método proposto.

PALAVRAS-CHAVE: Sistemas a Eventos Discretos, Identificação de Sistemas, Álgebra Max-plus, Grafos de Eventos Temporizados. 


\section{INTRODUÇÃO}

Diversas tecnologias presentes em processos de manufatura, redes de comunicação, controle de tráfego aéreo, etc., podem ser descritas por modelos com algumas características em comum. Nestes sistemas, o espaço de estados é em geral discreto (em muitos casos finito) e mudanças de estado acontecem somente em resposta à ocorrência de eventos. Essas características os distinguem dos sistemas que tem sua dinâmica dirigida pelo tempo e seu espaço de estado contínuo, cuja modelagem é tradicionalmente feita através das equações diferenciais e das equações a diferenças. Tais sistemas são denominados Sistemas a Eventos Discretos (SED) (Cassandras e Lafortune, 1999). Uma maneira formal de descrever SED é através de Redes de Petri (Murata, 1989) sendo as Redes de Petri temporizadas particularmente úteis quando há interesse em avaliar o desempenho do sistema.

Este artigo trata da abordagem Max-plus. Esta técnica fundamenta-se em resultados algébricos relativos aos semianéis idempotentes (também chamados de dióides) e na Teoria de Residuação, cujas bases são sumariadas na seção 2 . Sua principal característica é descrever a dinâmica de SED a partir de um sistema de equações algébricas lineares, escritas numa álgebra não convencional (Cohen et al., 1985). Quando SED apresentam fenômenos de sincronização de tarefas e de retardo no tempo, mas não há conflito na utilização de recursos, eles podem ser descritos pela álgebra Max-plus, sendo denominados SED Max-plus lineares (SEDMpl). Exemplos desses sistemas são as linhas de montagem em processos de manufatura. Conforme será discutido na seção 2, o comportamento desses tipos de SED coincide com o comportamento dos Grafos de Eventos Temporizados (GET) que são uma subclasse de Redes de Petri na qual todos os lugares têm uma única transição de entrada e uma única transição de saída (Baccelli et al., 1992). O uso dos GET como ferramenta gráfica facilita a compreensão da dinâmica dos SEDMpl.

Dois problemas centrais no estudo de SEDMpl são a identificação e o controle do sistema. O problema de controle é usualmente formulado dentro de um contexto just-in-time visando à minimizaçao dos estoques do sistema (Menguy et al. (2000b), Cottenceau et al. (2001), Lüders e SantosMendes (2002), Maia, Hardouin, Santos-Mendes e Cottenceau (2003)). O estudo da identificação de SEDMpl é abordado por Boimond et al. (1995) que propõem a identificação paramétrica baseada na resposta impulsiva do modelo. A abordagem considera a estimação de dois modelos ARMA: um para o regime transitório e outro para o comportamento periódico. Gallot et al. (1998) consideram a identificação a partir da resposta impulsiva do sistema baseada na decomposição do sistema em uma soma de sub-sistemas de primeira ordem (a resposta impulsiva é decomposta em uma soma de termos chamados elementos simples). Dessa forma o mé- todo consiste na estimação de parâmetros de diversos sistemas de primeira ordem. Menguy et al. (2000a) desenvolvem um algoritmo para a identificação não-paramétrica (direta) baseado no refinamento da estimativa da resposta impulsiva.

Este trabalho apresenta novos resultados (notadamente sobre as condições de excitação do sistema) para o método de identificação paramétrica de SEDMpl com uma entrada e uma saída (SEDMpl SISO) similar aos propostos por Maia, Santos-Mendes e Hardouin (2003) e Maia (2003). Nesse método supõem-se conhecidos o número de disparos durante o período transitório (i.e. o comprimento do transitório) e o número de disparos ocorridos em cada ciclo do regime permanente. Este último parâmetro está relacionado com o número de fichas no circuito crítico do GET. Por outro lado, são consideradas desconhecidas as durações temporais do transitório e do ciclo do regime permanente. Estes parâmetros devem ser estimados juntamente com os parâmetros que definem completamente a resposta transitória e o comportamento em regime do sistema. A principal contribuição em relação aos métodos propostos anteriormente é que, conhecida a estrutura do modelo, o modelo estimado é mais próximo do modelo real do sistema do que o obtido pelo cálculo direto da maior resposta impulsiva.

O artigo é organizado da maneira seguinte. A introdução às ferramentas matemáticas básicas para a compreensão do artigo é feita na seção 2 . O método de identificação é apresentado na seção 3, na qual os parâmetros supostos conhecidos e os parâmetros a serem estimados são formalmente explicitados. Na seção 4, são apresentados alguns exemplos ilustrativos e a conclusão é feita na seção 5.

\section{PRELIMINARES}

\subsection{Sistemas max-plus lineares}

Os resultados apresentados a seguir são extensivamente abordados em Baccelli et al. (1992).

Considere-se, a título de exemplo, o GET ilustrado pela figura 1, representando um sistema de montagem cuja produção máxima é de uma peça a cada duas unidades de tempo. Nessa figura $u$ é a transição de entrada à qual serão associados os instantes de admissão de matéria-prima ao sistema; $x_{i}$ são as transições internas (doravante também chamadas de estados) e $y$ é a transição de saída, que representa a conclusão do processo de montagem. Como é usual na literatura, a mesma notação será utilizada para designar uma transição de um GET e o instante de tempo de disparo (admissão de um produto em um dado setor do sistema) da transição. Dessa forma, o comportamento dinâmico do sistema é descrito pe- 


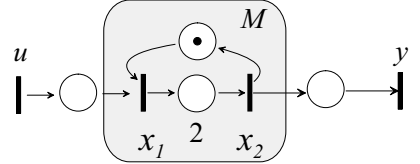

Figura 1: Descrição de um processo de montagem simples através de GET

las equações a seguir.

$$
\begin{aligned}
x_{1}(k) & =\max \left(x_{2}(k-1), u(k)\right) \\
x_{2}(k) & =2+x_{1}(k) \\
y(k) & =x_{2}(k) .
\end{aligned}
$$

A variável inteira $\mathrm{k}$ corresponde à numeração do disparo, convencionando-se que $\mathrm{k}=0$ corresponde ao primeiro disparo e $\mathrm{k}=\mathrm{n}$ ao $(\mathrm{n}+1)$-ésimo disparo.

Renomeando o operador max como sendo $\oplus$ e o operador + como sendo $\otimes$, pode-se reescrever:

$$
\begin{aligned}
x_{1}(k) & =x_{2}(k-1) \oplus u(k) \\
x_{2}(k) & =2 \otimes x_{1}(k) \\
y(k) & =x_{2}(k) .
\end{aligned}
$$

Tem-se portanto um sistema de equações recursivas lineares numa nova álgebra que é um semi-anel idempotente (ou dióide) e se caracteriza por um conjunto e duas operações (soma e produto), notado $(D, \oplus, \otimes)$, tal que a soma seja associativa, comutativa e idempotente $(a \oplus a=a)$, e o produto seja associativo (mas não necessariamente comutativo) e distributivo à esquerda e à direita em relação à soma. Além disso, devem existir elementos neutros para ambas as operações, notados por $\varepsilon$ (elemento nulo) e por $e$ (elemento unitário), e o elemento nulo deve ser absorvente em relação ao produto, isto é, $\forall a \in D, a \oplus \varepsilon=a ; a \otimes e=a ; a \otimes \varepsilon=\varepsilon$. Percebe-se que o conjunto $\mathbb{Z} \cup\{-\infty\}$ munido das duas operações $\oplus \equiv \max \mathrm{e} \otimes \equiv+$ é um dióide, no qual $\varepsilon=-\infty \mathrm{e}$ $e=0$. Num dióide uma relação de ordem é definida como $a \preceq b \Leftrightarrow b=a \oplus b$. Em geral, um dióide é completo se ele for fechado em relação a somas infinitas e se o produto for distributivo em relação a somas infinitas, sendo o seu maior elemento denotado por $T$ (topo). A operação $\wedge$ (ínfimo) é definida em $a \wedge b$ como sendo o maior elemento do dióide menor do que $a$ e menor do que $b$. A estrutura $(\mathbb{Z} \cup\{-\infty\} \cup\{\infty\}, \max ,+)$ é um dióide completo usualmente denominado "max-plus" e notado por $\overline{\mathbb{Z}}_{\max }$.

O exemplo mostrado na figura 1 utiliza o que se convenciona chamar de datadores, isto é, sequências crescentes $\{x(k)\}$ que representam as datas ou instantes de ocorrência dos disparos da transição $x$.
De modo análogo ao que ocorre na teoria de sistemas contínuos, as manipulações das equações de SEDMpl ficam facilitadas se forem utilizadas transformadas das sequências de datadores definidas de modo semelhante ao das clássicas transformadas Z. Define-se, portanto, a $\gamma$-transformada de uma sequência $\{x(k)\}$ como sendo $x(\gamma)=\bigoplus_{k \in \mathbb{Z}^{+}} x(k) \gamma^{k}$, sendo $\gamma$ uma variável abstrata cujo significado é o de um operador "atraso em contagem", pois é imediato constatar que $y(\gamma)=\gamma x(\gamma) \Leftrightarrow\{y(k)\}=\{x(k-1)\}$. As equações 1 , submetidas à $\gamma$-transformada resultam no seguinte sistema de equações algébricas:

$$
\begin{aligned}
x_{1}(\gamma) & =\gamma x_{2}(\gamma) \oplus u(\gamma) \\
x_{2}(\gamma) & =2 x_{1}(\gamma) \\
y(\gamma) & =x_{2}(\gamma)
\end{aligned}
$$

É possível definir operações de soma e multiplicação entre séries formais do tipo $\bigoplus_{k \in \mathbb{Z}^{+}} x(k) \gamma^{k}$, utilizando-se para isso a soma e o produto adotados para $\overline{\mathbb{Z}}_{\text {max }}$ combinados com os procedimentos usuais para somar e multiplicar séries formais. O conjunto de todas as séries formais em $\gamma$, munido dessas duas operações também é um dióide, isto é, satisfaz a todas as propriedades utilizadas para caracterizar um dióide, sendo denominado $\overline{\mathbb{Z}}_{\max } \llbracket \gamma \rrbracket$. A valoração, um conceito que será utilizado a seguir, de uma série $h$ em $\overline{\mathbb{Z}}_{\max } \llbracket \gamma \rrbracket$ é o menor expoente de coeficiente não-nulo, sendo representado por $\operatorname{val}(h)$.

A equação 2 pode ser colocada em forma matricial, resultando em $x(\gamma)=A(\gamma) x(\gamma) \oplus B(\gamma) u(\gamma)$ e $y(\gamma)=$ $C(\gamma) x(\gamma)$, sendo $A, B$ e $C$ matrizes na variável abstrata gama de dimensões apropriadas e $u(\gamma), y(\gamma)$ e $x(\gamma)$ vetores associados respectivamente às transições de entrada, de saída e internas ao sistema. De modo geral, em qualquer dióide, equações do tipo $x=a x \oplus b$ tem como solução mínima $x=a^{*} b$, sendo $a^{*}=\bigoplus_{i=0}^{\infty} a^{i}$ e $a^{0}=e$. Esse resultado, chamado de teorema da estrela ${ }^{1}$ (Baccelli et al., 1992), pode ser generalizado para as equações matriciais anteriores, levando a $y=C A^{*} B u$, ou de modo sintético:

$$
y(\gamma)=h(\gamma) u(\gamma)
$$

Nessa equação, $h(\gamma)=C A^{*} B$ é chamada de função de transferência do sistema e (como na teoria de sistemas contínuos) coincide com a resposta do sistema a uma entrada impulsiva, $u(\gamma)=e$, que corresponde a infinitos disparos da transição de entrada em $t=0$. Conforme mostrado em Baccelli et al. (1992) todo SEDMpl SISO tem uma resposta impulsiva periódica que pode ser colocada na seguinte forma:

$$
h(\gamma)=p(\gamma) \oplus q(\gamma) \gamma^{\nu}\left(s \gamma^{r}\right)^{*},
$$

\footnotetext{
${ }^{1}$ A operação * é a clássica estrela de Kleene utilizada em Teoria de Autômatos e Linguagens Formais.
} 
sendo $p(\gamma)=\bigoplus_{i=0}^{\nu-1} p_{i} \gamma^{i}, p_{i} \in \mathbb{N}$ e $q(\gamma)=\bigoplus_{j=0}^{r-1} q_{j} \gamma^{j}$, $q_{i} \in \mathbb{N}$ polinômios e $\nu \in \mathbb{N}, s \in \mathbb{N}$ e $r \in \mathbb{N}$ respectivamente o comprimento do transitório, a duração e o comprimento do regime permanente. $\mathrm{O}$ algoritmo de identificação proposto deverá, portanto, estimar os coeficientes dos polinômios $p(\gamma)$ e $q(\gamma)$ assim como o parâmetro $s$ (duração do ciclo do regime permanente $)^{2}$. Vale observar na equação 4 , que $h(i)=p_{i}$ para $(i<\nu)$ e que $h(j)=q_{i}$ para $(j<r)$. A figura 2 ilustra graficamente o comportamento de uma série periódica.

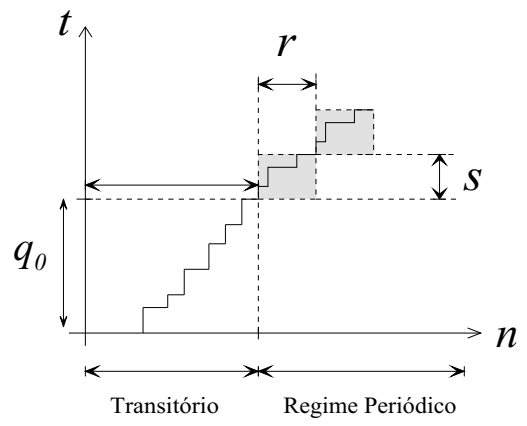

Figura 2: Comportamento da série $h(\gamma)$.

Em algumas situações pode ser interessante trabalhar no domínio dos datadores. Nesse domínio a equação 3 é escrita como

$$
y(k)=\bigoplus_{l=0}^{k} h(l) \otimes u(k-l) .
$$

Em outras palavras, isso significa que a saída é o resultado da convolução da entrada e da resposta ao impulso do GET. Esse resultado é obtido diretamente a partir da substituição da equação 4 na equação 3 .

\subsection{Teoria da residuação}

As formulações discutidas na próxima seção envolvem a inversão de funções, isto é, a solução em $x$ de equações do tipo $y=f(x)$. Diferentemente do caso da álgebra tradicional a solução desse tipo de equação no dióide pode apresentar um número infinito de soluções ou nenhuma solução. A Teoria da Residuação (Blyth e Janowitz, 1972) se ocupa justamente da solução deste problema em conjuntos parcialmente ordenados. A seguir, alguns resultados fundamentais relativos a essa teoria serão apresentados.

Consideram-se inicialmente os mapeamentos $f: \mathcal{D} \rightarrow \mathcal{E}$, sendo $\mathcal{D}$ e $\mathcal{E}$ dióides completos. Se $f$ for isotônico (preserva o ordenamento, i.e, $x \preceq y \Leftrightarrow f(x) \preceq f(y)$ ) e se existir um elemento máximo $x_{o p}$ do conjunto $\{x \mid y \succeq f(x), x \in$

\footnotetext{
${ }^{2}$ Observa-se que os graus dos polinômios $p(\gamma)$ e $q(\gamma)$ são respectivamente $\nu-1$ e $r-1$.
}

$\mathcal{D}, y \in \mathcal{E}\}$, diz-se que $x_{o p}$ é o resíduo do mapeamento $f$ em $y$. Se $f$ tiver um resíduo em qualquer ponto $y \in \mathcal{E}$, o mapeamento $f$ é dito residuável, sendo o resíduo denotado por $f^{\sharp}(y)$ (Baccelli et al., 1992). Dualmente, o mínimo do conjunto $\{x \mid y \preceq f(x), x \in \mathcal{D}, y \in \mathcal{E}\}$, se ele existir, é denominado resíduo dual sendo denotado por $f^{b}(y)$ e o mapeamento $f$ é dito dualmente residuável.

As funções $L_{a}(x)=a \otimes x$ e $R_{a}(x)=x \otimes a$ são residuáveis, sendo seus resíduos denotados respectivamente por $L_{a}^{\sharp}=a \phi$ e $R_{a}^{\sharp}=x \phi a$. No caso particular do dióide $\overline{\mathbb{Z}}_{\text {max }}$ tem-se $L_{a}^{\sharp}=$ $R_{a}^{\sharp}=x-a$ (operação de subtração da álgebra tradicional). A definição de residuação assegura que:

$$
a(a ф x) \preceq x .
$$

A função $T(x)=x \oplus a$ é dualmente residuável, sendo seu resíduo dual denotado por $x \ominus a$. Por exemplo, no dióide $\overline{\mathbb{Z}}_{\max }, 3 \ominus 1=3$, pois, pela definição de resíduo dual, $3 \ominus 1$ é a menor solução da equação $\max (x, 1) \geq 3$.

Conforme visto anteriomente, o dióide $\overline{\mathbb{Z}}_{\max } \llbracket \gamma \rrbracket$ é importante para a descrição da dinâmica de um GET. Algumas propriedades das séries descritas nesse dióide, fundamentais para este artigo, são apresentadas a seguir.

Propriedade 1 (Maia (2003)) O datador associado à série (trajetória não decrescente) $w(\gamma)=y(\gamma) \ominus x(\gamma)$ é dado por $w(k)=\bigoplus_{i \leq k}(y(i) \ominus x(i))$.

Lema 2 (Residuação de séries) Sejam duas séries do dióide $\overline{\mathbb{Z}}_{\max } \llbracket \gamma \rrbracket, u(\gamma)=\bigoplus_{k \in \mathbb{Z}^{+}} u(k) \gamma^{k}$ e $v(\gamma)=$ $\bigoplus_{k \in \mathbb{Z}^{+}} v(k) \gamma^{k}$, então:

$$
v(\gamma) \phi u(\gamma)=\bigoplus_{k \in \mathbb{Z}^{+}} \bigwedge_{l \in \mathbb{Z}^{+}}(v(l) \phi u(l+k)) \gamma^{k}
$$

Vale dizer que a expressão $\bigwedge_{l \in \mathbb{Z}^{+}}(v(l) \phi u(l+k))$ corresponde ao coeficiente de $\gamma^{k}$, sendo $(\bigwedge)$ o operador $\min ^{3}$ e $v(l) \phi u(l+$ $k)=u(l+k)-v(l)$ (operação de subtração da álgebra tradicional). Convém ainda relembrar a definição de residuação, que assegura que $y(\gamma)=v(\gamma) \phi u(\gamma)$ é a maior série tal que $v(\gamma) \otimes y(\gamma) \preceq u(\gamma)$. Maiores detalhes sobre a residuação de séries são apresentados por Baccelli et al. (1992). Programas para a manipulação de séries na álgebra de dióides usando o pacote computacional SCILAB podem ser encontrados em http://www.istia-angers.fr/ hardouin/outils.html.

\section{MÉTODO DE IDENTIFICAÇÃO}

No desenvolvimento do método proposto, assume-se que um modelo para o sistema existe e é expresso pela equação 3 .

\footnotetext{
${ }^{3} a \wedge b$ é o maior elemento menor que $a$ e menor que $b$.
} 
A estrutura, i.e. parâmetros $\nu$ e $r$ (equação 4 ), é também suposta conhecida. O objetivo do método de identificação é, então, estimar os polinômios desconhecidos $p(\gamma), q(\gamma)$ e a duração do período $s$. Mostra-se (Maia, 2003) que esse sistema pode ser representado pelas equações seguintes no dióide $\overline{\mathbb{Z}}_{\text {max }}$ :

$$
\begin{aligned}
z(k)= & s \otimes z(k-r) \oplus u(k) \\
y(k)= & p_{0} u(k) \oplus \ldots \oplus p_{\nu-1} u(k-\nu+1) \oplus \\
& q_{0} z(k-\nu) \oplus \ldots \oplus q_{r-1} z(k-\nu-r+1)
\end{aligned}
$$

com condições iniciais tais que $z(k)=u(k)=y(k)=\varepsilon$ para $k<0$, sendo $z(k)$ uma variável que modela o comportamento do ciclo.

Seguindo o mesmo raciocínio clássico da teoria de identificação para sistemas dinâmicos contínuos (Ljung, 1987), $y(k)$ pode ser reescrito como:

$$
y(k)=\varphi_{k}^{T} \otimes \theta,
$$

sendo $\varphi_{k}^{T}=[u(k) \ldots u(k-\nu+1) z(k-\nu) \ldots z(k-\nu-r+1)]$ o vetor de regressão e $\theta=\left[p_{0} \ldots p_{\nu-1} q_{0} \ldots q_{r-1}\right]^{T}$ o vetor de parâmetros a ser estimado.

Dessa forma, a partir da observação de $\mathrm{N}$ disparos das transições de saída e de entrada, obtém-se a equação matricial:

$$
Y=\Phi \otimes \theta
$$

na qual $\Phi=\left[\varphi_{0} \ldots \varphi_{N}\right]^{T}$ é a matriz de regressão e $Y=$ $[y(0) \ldots y(N)]^{T}$ é o vetor de saída observado.

Para estimação do parâmetro $\theta$, é conveniente a definição de um critério de erro como

$$
J(\widetilde{\theta})=\bigoplus_{k}(y(k)-\widetilde{y}(k))
$$

sendo a saída do modelo estimado $(\widetilde{y}(k)=\Phi \otimes \widetilde{\theta})$ tal que $\widetilde{y}(k) \leq y(k)$. Esse critério significa que, para uma mesma entrada, o melhor modelo deve produzir a maior saída possível que seja menor que a saída observada, i.e., deve-se escolher o maior $\widetilde{\theta}$ tal que $\Phi \otimes \widetilde{\theta} \preceq Y$.

Por enquanto, apenas para o desenvolvimento do algoritmo, assume-se que a variável $z$ é conhecida. Assim, um estimador ótimo para o critério $J(\widetilde{\theta})$ é obtido diretamente através da Teoria da Residuação,

$$
\widehat{\theta}=\bigoplus_{\Phi \otimes \widetilde{\theta} \preceq Y} \tilde{\theta}=\Phi \phi Y .
$$

Explicitamente, a solução dessa equação é dada por:

$$
\begin{aligned}
\widehat{p_{i}}=\bigwedge_{k=0}^{N} u(k-i) \phi y(k), & i \in\left[\begin{array}{ll}
0 & \nu-1
\end{array}\right], \\
\widehat{q_{j}}=\bigwedge_{k=0}^{N} z(k-\nu-j) \phi y(k), & j \in\left[\begin{array}{ll}
0 & r-1
\end{array}\right] .
\end{aligned}
$$

Nota-se que $\widehat{p}_{i} \geq p_{i}$ e $\widehat{q}_{j} \geq q_{j}$ pois $\widehat{\theta}$ é a maior solução de $\Phi \otimes \widetilde{\theta} \preceq Y$. Conseqüientemente, $\widehat{\theta}$ é uma solução para a equação (10), i.e., $Y=\Phi \otimes \widehat{\theta}$. Esse resultado implica que $\widehat{p}_{i}$ e $\widehat{q}_{j}$ satisfazem a equação (8) para $k=1, \ldots, N$. Atribuindo $u(k)=y(k)=\top$ para $k>N$ (isso significa que nenhum evento ocorre para $k>N$ ), a equação (8) é satisfeita para todo $k \in \mathbb{Z}$. Dessa maneira, a aplicação da transformação $\gamma$ resulta em

$$
y(\gamma)=\left[\hat{p}(\gamma) \oplus \hat{q}(\gamma) \gamma^{\nu}\left(s \gamma^{r}\right)^{*}\right] u(\gamma) .
$$

Propriedade 3 A condição necessária e suficiente para a convergência do estimador $\widehat{p}$ apresentado nas equações 13 é: $\forall i \in\left[\begin{array}{ll}0 & \nu-1\end{array}\right], \exists k^{\prime} \in\left[\begin{array}{ll}0 & N\end{array}\right]$ tal que $y\left(k^{\prime}\right)=p(i)+u\left(k^{\prime}-i\right)$. Analogamente, para $\widehat{q}$ a condição necessária e suficiente é: $\forall j \in\left[\begin{array}{ll}0 & r-1\end{array}\right], \exists k^{\prime} \in\left[\begin{array}{ll}0 & N\end{array}\right]$ tal que $y\left(k^{\prime}\right)=q(j)+z(k-$ $\nu-j)$.

Prova: Como $\widehat{p}_{i}=\bigwedge_{k=0}^{N} u(k-i) \phi y(k)$, a necessidade é obtida lembrando-se que para $u(k-i)$ e $y(k)$ finitos tem-se $u(k-i) \oint y(k)=y(k)-u(k-i)$. A suficiência é obtida através do fato de que $\widehat{p}$ é máximo e de que $y\left(k^{\prime}\right)=p(i)+u\left(k^{\prime}-i\right)$ implica em $\widehat{p_{i}} \leq p_{i}$, ou seja, esses dois fatos resultam em $\widehat{p_{i}}=p_{i}$. Para $\widehat{q}$, a demonstração é análoga.

Contudo esse resultado tem pouca utilidade prática já que não explicita como deve ser a excitação do sistema para que a estimação seja correta. Uma condição de excitação suficiente é apresentada na proposição 5 .

Lema 4 Se a excitação do GET é tal que $0 \leq u(k) \leq$ $(h(\gamma) \oint h(\gamma))(k)$ para $0 \leq k \leq N$ então $y(k)=h(k)$.

Prova: Pela fórmula 5, $y(k)=\bigoplus_{l=0}^{k} h(l) \otimes u(k-$ l). Dessa forma, $y(k) \geq h(k)$ pois $u(i) \geq 0$ para $0 \leq i \leq N$. Por outro lado: $y(k) \leq$ $\bigoplus_{l=0}^{k} h(l)(h(\gamma) ф h(\gamma))(k-l)=\bigoplus_{l=0}^{k} h(l)\left(\bigwedge_{i \in \mathbb{Z}^{+}} h(i) \phi h(i+\right.$ $k-l)) \leq \bigoplus_{l=0}^{k} h(l)(h(l) ф h(k))$, pois o lema 2 , assegura que $(h(\gamma) \phi h(\gamma))(k-l)=\bigwedge_{i \in \mathbb{Z}^{+}} h(i) \phi h(i+k-l)$. Assim pela inequação 6, $\bigoplus_{l=0}^{k} h(l)(h(l) ф h(k)) \leq \bigoplus_{l=0}^{k} h(k)=h(k)$ $e$, portanto, $y(k) \leq h(k)$ para $0 \leq i \leq N$. Como já foi mostrado que $y(k) \geq h(k)$, conclui-se que $y(k)=h(k)$.

Como conseqüência desse lema, se $e \preceq u(\gamma) \preceq h(\gamma) \oint h(\gamma)$ então $y(\gamma)=h(\gamma)$, em outras palavras $h(\gamma) \oint h(\gamma)$ é a maior entrada tal que a saída seja igual à resposta ao impulso. A interpretação desse resultado para um sistema de manufatura é que $h(\gamma) ф h(\gamma)$ fornece os instantes máximos de disparo da transição de entrada $u(\gamma)$ de forma que a saída permaneça inalterada e igual a $h(\gamma)$. Em teoria de identificação de sistemas contínuos (Ljung, 1987), quando um sinal consegue 
excitar toda a faixa dinâmica de um sistema, diz-se que esse sinal é um "sinal de excitação rico" para o sistema. Analogamente, devido ao lema 4, uma sequiência de disparos da transição de entrada será considerada um "sinal de excitação rico" para um GET, se a condição $e \preceq u(\gamma) \preceq h(\gamma) \downarrow h(\gamma)$ for verificada.

Proposição 5 Se o parâmetro $s$ é conhecido e o sinal de entrada $u$ é suficientemente "rico" (i.e., $0 \leq u(k) \leq$ $(h(\gamma) \oint h(\gamma))(k)$ para $0 \leq k \leq N$ sendo $N \geq \nu+r-1)$ então os estimadores dados em 13 convergem para os parâmetros reais do sistema, precisamente $\widehat{p_{i}}=p_{i}$ e $\widehat{q_{j}}=q_{j}$ para $i \in\left[\begin{array}{ll}0 & \nu-1\end{array}\right]$ e $j \in\left[\begin{array}{ll}0 & r-1\end{array}\right]$.

Prova: As hipóteses desta proposição para o sinal de entrada implicam, de acordo com o lema 4, que $y(k)=h(k)$ para $0 \leq k \leq N$. Dessa forma, $\widehat{p}_{i}=\bigwedge_{k=0}^{N} u(k-i) \phi h(k) \leq$ $u(0) \oint h(i) \leq h(i)=p_{i}(i<\nu)$ pois $u(0) \geq 0$. Além disso, se $s$ é conhecido, então $z$ é também conhecido e os estimadores propostos sempre asseguram que $\widehat{p}_{i} \geq p_{i}$ e $\widehat{q}_{j} \geq$ $q_{j}$, pois eles são as maiores soluções para o problema. Assim, conclui-se que $\widehat{p}_{i}=p_{i}$. O mesmo raciocínio é aplicado para a estimativa $\widehat{q}_{j}$ lembrando que $q_{j}=h(\nu+j)$ para $j<r$.

Convém observar que se $u(k)-u(0) \leq(h(\gamma) ф h(\gamma))(k)$, o estimador também converge. De fato, fazendo $u(k)=$ $\bar{u}(k)+u(0)=\bar{u}(k) \otimes u(0)$, então $y(k)=\bar{y}(k) \otimes u(0)$ sendo $\bar{y}(k)=\bigoplus_{l=0}^{k} h(l) \otimes \bar{u}(k-l)$. Do mesmo modo, mostra-se que $z(k)=\bar{z}(k) \otimes u(0)$. Dessa forma, a diferença entre os datadores $(u, y, z)$ e $(\bar{u}, \bar{y}, \bar{z})$ é o termo $u(0)$. Como os resíduos das equações 13 são calculados a partir de diferenças entre datadores, o resultado obtido utilizando $(u, y, z)$ é mesmo que se obtém utilizando $(\bar{u}, \bar{y}, \bar{z})$.

Nota 6 Se $u\left(k_{1}\right)>>u\left(k_{1}-1\right)$ para $k_{1}>0$, então $y\left(k_{1}+j\right)=u\left(k_{1}\right) \otimes\left(\bigoplus_{l=0}^{j} h(j-l) \otimes \bar{u}(l)\right)$ para $j \geq 0$, sendo $u\left(k_{1}+j\right)=u\left(k_{1}\right)+\bar{u}(j)=u\left(k_{1}\right) \otimes \bar{u}(j)$. Esse resultado permite escrever a saída como $y\left(k_{1}+j\right)=u\left(k_{1}\right) \otimes \bar{y}(j)$ sendo $\bar{y}$ a saída do sistema para a entrada $\bar{u}$, ou seja, a partir do $k_{1}$ ésimo disparo, o sistema desconsidera a influência dos disparos anteriores. Do mesmo modo, a variável interna é escrita como $z\left(k_{1}+j\right)=u\left(k_{1}\right) \otimes \bar{z}(j)$ sendo $\bar{z}$ a trajetória obtida utilizando a entrada $\bar{u}$. Como conseqüência dessa observação, se $\bar{u}(i) \leq(h(\gamma) \phi h(\gamma))(i)$ para $0 \leq i \leq \nu+r-1$, então os estimadores também convergem conforme observado anteriormente. De fato, sabe-se que:

$y\left(k_{1}+j\right)=\bigoplus_{i=0}^{k_{1}-1}\left(h\left(k_{1}+j-i\right) \otimes u(i)\right) \oplus \bigoplus_{i=k_{1}}^{k_{1}+j}\left(h\left(k_{1}+\right.\right.$ $j-i) \otimes u(i))$.

Como $u$ é crescente e $u\left(k_{1}\right)>>u\left(k_{1}-1\right)$ então o segundo somatório é maior que o primeiro. Assim, como $u\left(k_{1}+j\right)=$ $u\left(k_{1}\right) \otimes \bar{u}(j)$, tem-se que:

$$
y\left(k_{1}+j\right)=u\left(k_{1}\right) \otimes\left(\bigoplus_{i=k_{1}}^{k_{1}+j} h\left(k_{1}+j-i\right) \otimes \bar{u}\left(i-k_{1}\right)\right) .
$$

Fazendo $l=i-k_{1}$, obtém-se que $y\left(k_{1}+j\right)=$ $u\left(k_{1}\right)\left(\bigoplus_{l=0}^{j} h(j-l) \otimes \bar{u}(l)\right)$. Seguindo o mesmo argumento, mostra-se que $z\left(k_{1}+j\right)=u\left(k_{1}\right) \otimes \bar{z}(j)$ sendo $\bar{z}$ a trajetória obtida utilizando a entrada $\bar{u}$.

Uma interpretação para a situação apresentada na nota 6 é que após o $\left(k_{1}-1\right)$-ésimo disparo da transição de entrada espera-se um tempo tal que o sistema retorne à condição de "relaxado" ${ }^{4}$ para só assim disparar novamente essa transição. Dessa forma, a menos da translação temporal $u\left(k_{1}\right)$, o sistema passa a responder a partir do $k_{1}$-ésimo disparo como se estivesse no início do processo.

Propriedade 7 Para o estimador proposto tem-se sempre $\widehat{p_{0}}=p_{0}$, ou seja, $\widehat{p_{0}}$ sempre converge para o valor real independentemente das condições de excitação do sistema.

Prova: Isso se deve o fato de que $\widehat{p_{0}}=\bigwedge_{k=0}^{N} u(k) \phi y(k) \leq$ $u(0) \phi y(0)=y(0)-u(0)$. Como $y(0)=p_{0} \otimes u(0)=$ $p_{0}+u(0)$, então $\widehat{p_{0}} \leq p(0)$. Entretanto, como discutido anteriormente, $\widehat{p}_{0} \geq p_{0}, \operatorname{logo} \widehat{p}_{0}=p_{0}$.

A estimação dos parâmetros $q_{j}$ apresentada nas equações 13 requer o conhecimento da variável $z$. Essa variável é desconhecida mas pode ser estimada. Se uma estimativa de $s$ (representada por $\widehat{s}$ ) é disponível, então uma estimativa de $z$ (representada por $\widehat{z}$ ) é obtida iterativamente segundo a equação (8), ou seja,

$$
\widehat{z}(k)=\widehat{s} \otimes \widehat{z}(k-r) \oplus u(k) .
$$

Dessa forma, estima-se a variável $z$ a partir da estimativa de $s$. Para a estimativa da duração do período, $s$, deve-se recordar que as estimativas dadas na equação 13 devem satisfazer a equação 14. A partir desse resultado, demonstra-se a proposição 8 .

Proposição 8 Um limitante superior de $s$ é calculado a partir das expressões abaixo:

$$
\begin{aligned}
w_{\text {inf }}(\gamma) & =y(\gamma) \ominus \widehat{p}(\gamma) u(\gamma) \\
c(\gamma) & =w_{\text {inf }}(\gamma) \phi y(\gamma) \\
s_{u p} & =\min _{i=1, \ldots, L}\left\lfloor\frac{c(i r)}{i}\right\rfloor,
\end{aligned}
$$

\footnotetext{
${ }^{4} \mathrm{O}$ sistema é dito relaxado num determinado instante de tempo se nenhum disparo puder ocorrer, a partir desse instante, nas transições internas e de saída do sistema, supondo que não ocorra nenhum disparo nas transições de entrada do sistema.
} 
sendo $L=\left\lfloor\frac{N}{r}\right\rfloor$ e $u(\gamma)$ e $y(\gamma)$ respectivamente ${ }^{5}$ as séries correspondentes aos dados da entrada e da saída do sistema.

Prova: A demonstração é apresentada por Maia (2003) e utiliza os resultados da propriedade 1 e do lema 2.

Dessa forma, utiliza-se neste artigo o estimador para $s$ como sendo $\widehat{s}=s_{\text {up }}$.

Uma condição suficiente para a convergência do estimador proposto é apresentada a seguir. Convém lembrar que para que os efeitos da periodicidade da função de transferência sejam percebidos na saída é sempre necessário pelo menos $\nu+r$ observações dos disparos da transição de saída (ver figura 2).

Proposição 9 (Maia (2003)) Sejam $w_{\text {inf }}(\gamma) \neq \varepsilon, v_{w}=$ $\operatorname{val}\left(w_{\text {inf }}(\gamma)\right)$ e o número de observações tal que $N \geq r+v_{w}$. Se $0 \leq u(k) \leq(h(\gamma) \phi h(\gamma))(k)$ para $0 \leq k \leq N$, então $\widehat{s}=s$.

Vale ressaltar que a condição de convergência apresentada na proposição 9 é ampliada se o sinal de entrada satisfaz às hipóteses apresentadas na nota 6 para $k_{1} \leq k \leq k_{1}+N$ e $v_{w} \geq k_{1}$.

Finalmente, o método de identificação é sintetizado no algoritmo mostrado a seguir.

\section{Algoritmo}

\section{begin}

Iniciar variáveis: $z(k)=u(k)=y(k)=-\infty$ para $k<0$.

Coletar $N \geq \nu+r$ pares de dados de entrada e saída $(u(k), y(k))$;

$\widehat{p}_{i}=\bigwedge_{k=0}^{N} u(k-i) \phi y(k) i=0, \ldots, \nu-1 ;$

for $k=0, \ldots, N$

$$
\begin{aligned}
& (\widehat{p}(\gamma) u(\gamma))(k)=\bigoplus_{i=0}^{\nu-1}\left(\widehat{p}_{i} \otimes u(k-i)\right) ; \\
& w_{i n f}(k)=\bigoplus_{i=0}^{k}\{y(i) \ominus(\widehat{p}(\gamma) u(\gamma))(i)\} ;
\end{aligned}
$$

end

$c(k)=\bigwedge_{i=0}^{N-k} w_{i n f}(i) \phi y(k+i)$ for $k=0, \ldots, N ;$

$\widehat{s}=\min _{i=1, \ldots, L}\left\lfloor\frac{c(i r)}{i}\right\rfloor$ sendo $L=\left\lfloor\frac{N}{r}\right\rfloor ;$

$\widehat{z}(k)=\widehat{s} \otimes \widehat{z}(k-r) \oplus u(k)$ for $k=0, \ldots, N ;$

\footnotetext{
${ }^{5}$ Convenciona-se que $\lfloor x\rfloor$ é o maior inteiro menor que $x$.
}

$\widehat{q_{j}}=\bigwedge_{k=0}^{N} \widehat{z}(k-\nu-j) \phi y(k)$ for $j=0, \ldots, r-1 ;$

end

\section{EXEMPLOS ILUSTRATIVOS}

A figura 3 representa um GET que modela um sistema de montagem com 3 máquinas representadas pelos símbolos $M_{1}, M_{2}$ e $M_{3}$. Sejam $u$ e $y$ respectivamente os datadores das transições de entrada e de saída e $x_{1}, x_{2}$ e $x_{3}$ os datadores das transições internas desse GET. Utilizando a transforma-

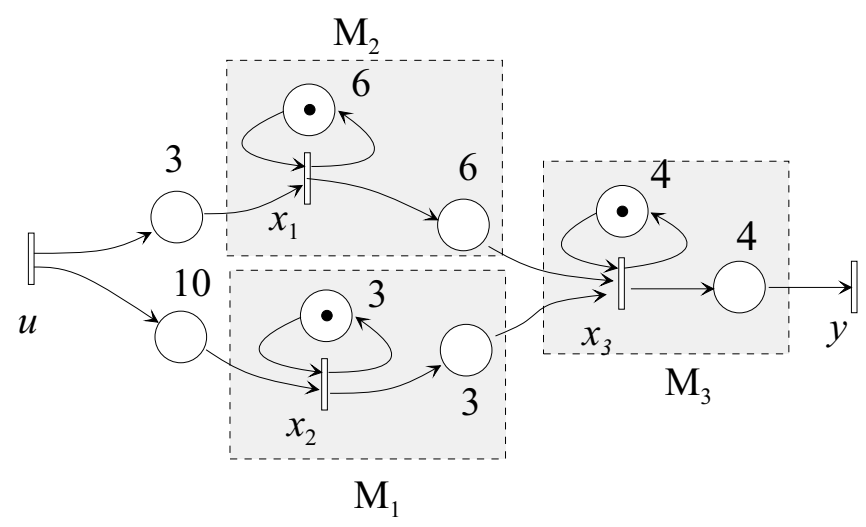

Figura 3: Exemplo de Grafo de Eventos Temporizados SISO

ção $\gamma$, obtém-se o sistema de equações (16) que relaciona essas variáveis no dióide $\overline{\mathbb{Z}}_{\max } \llbracket \gamma \rrbracket$.

$$
\begin{array}{ccc}
x_{1}(\gamma) & = & 6 \gamma x_{1}(\gamma) \oplus 3 u(\gamma) \\
x_{2}(\gamma) & = & 3 \gamma x_{2}(\gamma) \oplus 10 u(\gamma) \\
x_{3} \gamma & = & 4 \gamma x_{3}(\gamma) \oplus 6 x_{1}(\gamma) \oplus 3 x_{2}(\gamma) \\
y(\gamma) & = & 4 x_{3}(\gamma)
\end{array}
$$

A partir do teorema da estrela apresentado na introdução, obtém-se a equação que relaciona a entrada e a saída do sistema

$$
y(\gamma)=\left(17 \oplus 21 \gamma \oplus\left(25 \gamma^{2}\right)(6 \gamma)^{*}\right) u(\gamma)
$$

sendo $h(\gamma)=17 \oplus 21 \gamma \oplus\left(25 \gamma^{2}\right)(6 \gamma)^{*}=17 \oplus 21 \gamma \oplus$ $\left.25 \gamma^{2} \oplus 31 \gamma^{3} \oplus 37 \gamma^{5} \oplus 43 \gamma^{5} \ldots\right]$ a função de transferência que apresenta parâmetros estruturais $\nu=2 \mathrm{e} r=1$. Em outras palavras, a seqüência de disparos da resposta impulsiva é $h=\left[\begin{array}{llllll}17 & 21 & 25 & 31 & 37 & 43 \ldots\end{array}\right]$.

A planta descrita pela equação 17 será utilizada a seguir em diversas condições de excitação para ilustrar a aplicabilidade do método de identificação proposto. Nesse caso, a máxima entrada que garante a "excitação rica" do sistema é $u_{h}(\gamma)=$ $h(\gamma) \phi h(\gamma)=0 \oplus 4 \gamma \oplus 8 \gamma^{2} \oplus 14 \gamma^{3} \oplus 20 \gamma^{5} \oplus 26 \gamma^{5} \ldots$, ou seja,

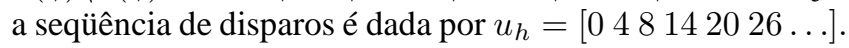


Em cada exemplo, o par de entrada e saída $\left(u_{h}, h\right)$ é mostrado nos gráficos em linhas pontilhadas.

Exemplo 10 Considere-se a seqüência de disparos da transição de entrada dada por $u=\left[\begin{array}{llllll}0 & 2 & 5 & 8 & 17 & 20\end{array}\right]$. Conseqüentemente, a seqüência de disparos da transição de saída é

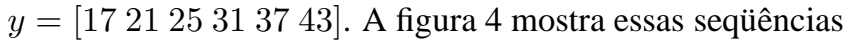
e o comportamento do GET em linhas pontilhadas quando a entrada é $u_{h}$. Nesse caso, observa-se que $u \preceq u_{h}$ e que a condição de excitação do sistema satisfaz a hipótese da proposição 9. Como consequiência, a aplicação do método de identificação proposto resulta em $\widehat{p}_{0}=17, \widehat{p}_{1}=21, \widehat{q}_{0}=25$ e $\widehat{s}=6$. Isto é, o método converge para os parâmetros reais do sistema.

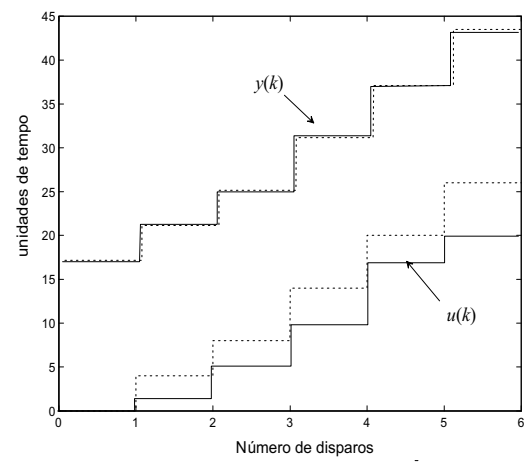

Figura 4: Comportamento do GET para o exemplo 10

Exemplo 11 Neste exemplo, supõe-se que a seqüência de disparos da transição de entrada seja dada por $u=$

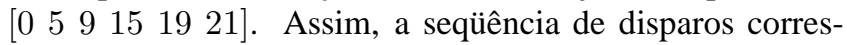

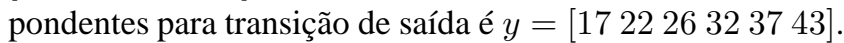
Esses dados são mostrados na figura 5. A aplicação do método de identificação proposto resulta em $\widehat{p}_{0}=17, \widehat{p}_{1}=21$, $\widehat{q}_{0}=25$ e $\widehat{s}=6$. Isto é, o método converge para os parâmetros reais do sistema embora a condição de excitação não satisfaça a hipótese da proposição 9 (i.e. $u \npreceq u_{h}$ ). Esse exemplo mostra que a proposição é suficiente para convergência mas não necessária.

Exemplo 12 Um outro exemplo de simulação utiliza uma seqüência de disparos da transição de entrada dada por $u=$

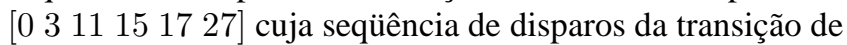

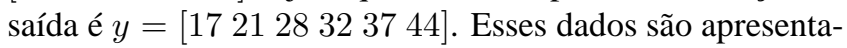
dos na figura 6. Neste exemplo a condição de excitação é tal que $u \npreceq u_{h}$ e os resultados obtidos pelo método de identificação são: $\widehat{p}_{0}=17, \widehat{p}_{1}=24, \widehat{q}_{0}=\varepsilon$ e $\widehat{s}=\top$. Nesse caso, os parâmetros não convergiram para os valores reais. Podese fazer uma analogia dessa situação com o caso de excitação "pobre" em sistemas dinâmicos contínuos.

Exemplo 13 A figura 7 mostra outra simulação para uma

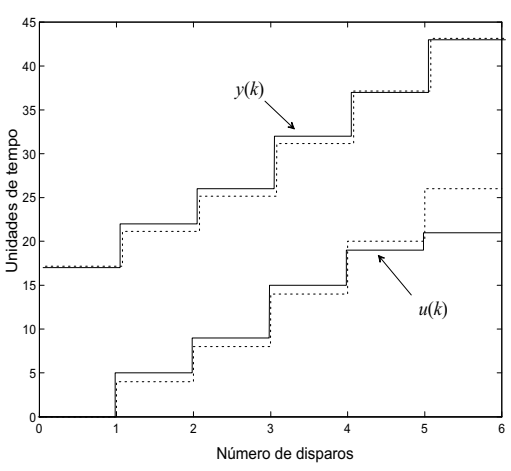

Figura 5: Comportamento do GET para o exemplo 11

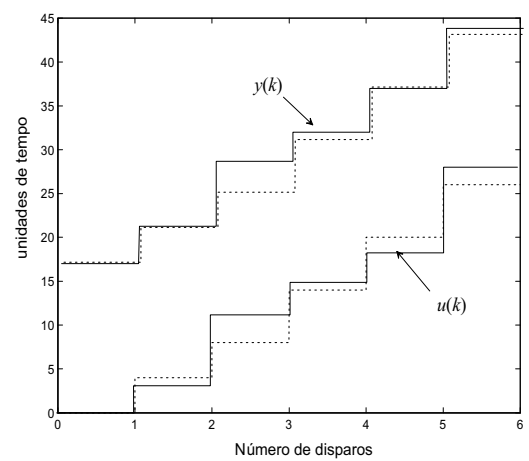

Figura 6: Comportamento do GET para o exemplo 12

seqüência de disparos da transição de entrada dada por $u=$

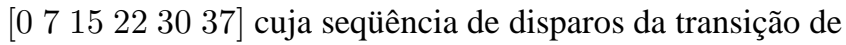

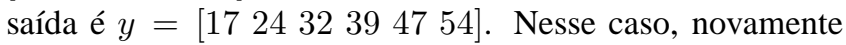
a condição de excitação não é satisfeita $\left(u \succeq u_{h}\right)$. Os resultados obtidos pelo método de identificação são: $\widehat{p}_{0}=17$, $\widehat{p}_{1}=24, \widehat{q}_{0}=\varepsilon$ e $\widehat{s}=\top$, isto é, os parâmetros não convergiram para parâmetros reais da planta.

Exemplo 14 Nesta simulação a entrada é dada por $u=$ $\left[\begin{array}{llllll}0 & 26 & 30 & 33 & 33 & 33\end{array}\right]$ e a saída correspondente é $y=$ [17 43475157 63]. A figura 8 mostra essas seqüências. Nesse caso, observar que $u \succeq u_{h}$. Como aconteceu no exemplo $11, \widehat{p}_{0}=17, \widehat{p}_{1}=21, \widehat{q}_{0}=25$ e $\widehat{s}=6$, ou seja, mesmo não satisfazendo a condição suficiente de excitação, os resultados obtidos mostram que estimadores convergem para os parâmetros reais do modelo da planta.

Os exemplos 12 e 14 ilustram o fato que se a excitação $u_{1}$ leva a uma estimação não-convergente então a excitação $u_{2} \succeq u_{1}$ não necessariamente leva a uma estimação não convergente. Esse ponto pode ser compreendido com o auxílo na nota 6.

O exemplo 13 ilustra particularmente o fato de que se incre- 


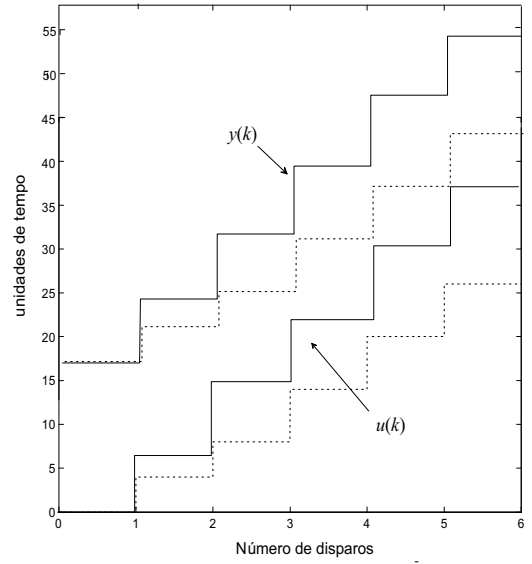

Figura 7: Comportamento do GET para o exemplo 13

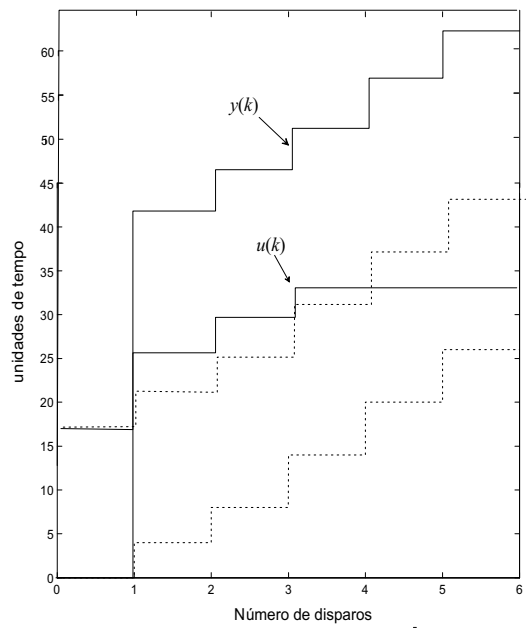

Figura 8: Comportamento do GET para o exemplo 14

mentos na trajetória de $u$ são muito grandes em relação aos de $h$, ou seja, a inclinação de $u$ é suficientemente maior que a de $h$, o algoritmo de estimação sempre resulta em $q_{j}=\varepsilon$ e $s=\top$. Isso se deve ao fato de que se $u(k)-u(k-1)$ é suficientemente grande para todo $k \in \mathbb{N}$ então as desigualdades $h(0) \otimes u(k) \succeq h(1) \otimes u(k-1) \succeq \ldots$ são verdadeiras. Como $y(k)=\bigoplus_{l=0}^{k} h(l) \otimes u(k-l)$ e pela equação $4, p_{i}=h(i)$ para $i \in\left[\begin{array}{ll}0 & \nu-1\end{array}\right]$, então essas desigualdades permitem escrever que $y(k)=\bigoplus_{l=0}^{\nu-1} p(l) \otimes u(k-l)$. Dessa forma $\widehat{p}$ é tal que $y(k)=\bigoplus_{l=0}^{\nu-1} \widehat{p}_{l} \otimes u(k-l)$, ou seja, $\widehat{p}$ é um modelo que assegura que o critério de erro é nulo. Intuitivamente, esse fato corresponde à situação em que a entrada de matéria-prima no sistema é lenta ao ponto de só se observarem fenômenos transitórios, isto é, a taxa de chegada de matéria-prima não permite que o polinômio $q(\gamma)$ influencie na solução. Dessa forma, segundo o método de estimação apresentado no algo-
Tabela 1: Condições de convergência observadas para os exemplos

\begin{tabular}{|c|c|c|}
\hline Exemplo & Excitação & Comportamento observado \\
\hline 10 & $u \preceq u_{h}$ & convergência \\
11 & $u \npreceq u_{h}$ & convergência \\
12 & $u \npreceq u_{h}$ & não-convergência \\
13 & $u \succeq u_{h}$ & não-convergência \\
14 & $u \succeq u_{h}$ & convergência \\
\hline
\end{tabular}

ritmo $1, w_{\text {inf }}(\gamma)(k)=\varepsilon$ e, conseqüentemente, $c(\gamma)(k)=\top$ para todo $k \in \mathbb{N}$. Finalmente, isso resulta em $\widehat{s}=\top$ e, para um número de observações $N \geq 2 r+\nu-1$, tem-se que $q_{j}=\varepsilon$ para $j \in\left[\begin{array}{ll}0 & r-1\end{array}\right]$.

A tabela 1 resume as condições de convergência observadas para os exemplos apresentados.

\section{CONCLUSÃO}

Este artigo apresentou alguns resultados para a estimação dos parâmetros temporais para SED Max-plus lineares que são uma extensão do trabalho apresentado por Maia, SantosMendes e Hardouin (2003). O método se baseia no conhecimento do comprimento do transitório e do comprimento do ciclo em regime permanente (respectivamente, parâmetros $\nu$ e $r$ ) e visa à estimação das durações do transitório e do ciclo em regime permanente (respectivamente, parâmetros $q_{o}$ e $s$ ) assim como a estimação dos demais coeficientes do polinômios $p(\gamma)$ e do polinômio $q(\gamma)$. As principais contribuições são relativas às condições de excitação do sistema que asseguram a convergência do método. Vale dizer que o modelo desenvolvido para a estimação utiliza uma variável interna desconhecida para modelar o comportamento do circuito crítico e a estimação dessa variável interna apresenta dificuldades e constitui uma limitação para o método, devendo portanto ser objeto de atenção em trabalhos futuros. Outro resultado apresentado é a determinação de um majorante para a duração do ciclo em regime permanente (parâmetro $s$ ) mesmo que as condições suficientes de excitação definidas pela proposição 9 não sejam respeitadas.

\section{REFERÊNCIAS}

Baccelli, F., Cohen, G., Olsder, G. e Quadrat, J. (1992). Synchronisation and Linearity: An Algebra for Discrete Event Systems, John Wiley and Sons, New York.

Blyth, T. e Janowitz, M. (1972). Residuation Theory, Pergamon Press, Oxford.

Boimond, J., Hardouin, L. e Chiron, P. (1995). A Modeling Method of SISO Discrete-Event Systems in MaxAlgebra, ECC'95, Rome, Italy, pp. 2023-2026. 
Cassandras, C. G. e Lafortune, S. (1999). Introduction to Discrete Event Systems, Kluwer Academic Publishers.

Cohen, G., Dubois, D., Quadrat, J. e Viot, M. (1985). A linear system theoretic view of discrete event processes and its use for performance evaluation in manufacturing, IEEE Trans. on Automatic Control AC-30: 210220.

Cottenceau, B., Hardouin, L., Boimond, J. e Ferrier, J. (2001). Model Reference Control for Timed Event Graphs in Dioid, Automatica 37: 1451-1458.

Gallot, F., Boimond, J. e Hardouin, L. (1998). Identification of Linear Systems using MA and ARMA Models in Dioids, IFAC Conference Structure and Control, Nantes, France.

Ljung, L. (1987). System Identification: Theory for the User, Prentice Hall.

Lüders, R. e Santos-Mendes, R. (2002). Generalized Multivariable Control of Discrete Event Systems in Dioid, 6th International Workshop on Discrete Event Systems (WODES'02), Zaragoza, Spain.

Maia, C. A. (2003). Identificação e Controle de Sistemas a Eventos Discretos na Álgebra (max,+), Tese de doutorado, UNICAMP, Campinas, SP, Brasil.

Maia, C. A., Hardouin, L., Santos-Mendes, R. e Cottenceau, B. (2003). Optimal Closed-loop of Timed Event Graphs in Dioids, IEEE Trans. on Automatic Control 48(12): 2284-2287.

Maia, C. A., Santos-Mendes, R. e Hardouin, L. (2003). Some Results on Identification of Timed Event Graphs in Dioids, 11th Mediterranean Conference on Control and Automation (MED'03), Rodes, Grécia.

Menguy, E., Boimond, J., Hardouin, L. e Ferrier, J. (2000a). A First Step Towards Adaptive Control for Linear Systems in Max Algebra, Discrete Event Dynamic Systems.Theory and Applications 10: 347-367.

Menguy, E., Boimond, J., Hardouin, L. e Ferrier, J. (2000b). Just-in-time Control of Timed Event Graphs Update of Reference Input, Presence of Uncontrollable Input, IEEE Trans. on Automatic Control 45(11): 2155-2158.

Murata, T. (1989). Petri nets : properties, analysis and applications., Proceedings of the IEEE 77(4): 541-580. 